that female $B R C A 1 / 2$ mutation carriers with at least one allele of 28 repeats or greater had an earlier age of onset of breast cancer than those with shorter alleles $^{7}$. Later studies did not support either of these findings, but most were limited to women with premenopausal breast cancer and did not examine the effect of CAG alleles of less than 20 repeats. A recent study that showed a strong protective effect of these short CAG alleles among postmenopausal women (where $5 \%$ of breast cancer cases and $15 \%$ of controls had at least one allele of less than 20 repeats) appeared to resolve the earlier paradoxical results: the choice of cutoffs of allele lengths and age at diagnosis have a crucial impact on the results ${ }^{8}$. Alas, the largest study of all, a nested case-control study within the Nurses' Health Study, finds no relationship whatsoever between short or long CAG alleles on breast-cancer risk in pre- or postmenopausal women ${ }^{9}$. Interestingly, we may not have reached the end of the story; the histopathology of the tumors has rarely been considered, and two studies have suggested that poorly differentiated breast cancer may be associated with short repeats ${ }^{10,11}$. Needless to say, larger, better-designed studies will be needed to confirm the result. So we are back to square one.

The mantra of 'larger, better-designed studies' seems to be the only riposte to the question, Where are all the genes? Maybe this will do the trick, but so far, for most candidate-gene studies, the relative risk tends towards 1.0 as sample size increases $^{12}$. Moreover, if alleles interact with each other and with environmental factors, one could envisage a situation where allele $\mathrm{A}$ is associated with breast cancer in population 1 , but not associated in population 2. This could seriously impede efforts to use genomic information to categorize individuals into meaningful risk groups.

Without some legitimate target candidates, enthusiasm for public health applications of genetic medicine may seem like Pollyanna's view of the world: irrepressible optimism but a certain amount of self-delusion. Only time will tell, but the work of Pharoah et al. clearly establishes the rules of the game.

1. Pharoah, P.D.P. et al. Polygenic susceptibility to breast cancer and implications for prevention. Nature Genet. 30 (2002); advance online publication, 4 March 2002 (DOI: 10.10138/ng853).

2. Antoniou, A.C. et al. Evidence for further breast cancer susceptibility genes in addition to BRCA1 and BRCA2 in a population-based study. Genet. Epidemiol. 21, 1-18 (2001).
3. Lichtenstein, P. et al. Environmental and heritable factors in the causation of cancer-analyses of cohorts of twins from Sweden, Denmark and Finland. N. Engl. J. Med. 343, 78-85 (2000).

4. Easton, D.F. How many more breast cancer predisposition genes are there? Breast Cancer Res. 1, 14-17 (1999).

5. Chenevix-Trench, G. et al. Dominant Negative ATM Mutations in Breast Cancer Families. J. Natl. Cancer Inst. 94, 205-215 (2002).

6. Elhaji, Y.A. et al. The polymorphic CAG repeat of the androgen receptor and female breast cancer. Am. J. Hum. Genet. 61S, abstract 64 (1997).

7. Rebbeck, T.R. et al. Modification of BRCA1-associated breast cancer risk by the polymorphic androgen receptor CAG repeat. Am. J. Hum. Genet. 64, 1371-1377 (1999).

8. Giguère, Y. et al. Short polyglutamine tracts in the androgen receptor are protective against breast cancer in the general population. Cancer Res. 61, 5869-5874 (2001)

9. Haiman, C.A. et al. The androgen receptor CAG repeat polymorphisms and risk of breast cancer in the Nurses' Health Study. Cancer Res. 62, 1045-1049 (2002).

10. Yu, H. et al. Shorter CAG repeat length in the androgen receptor gene is associate with more aggressive forms of breast cancer. Breast Cancer Res. Treat. 59, 153-161 (2000).

11. Elhaji, Y.A. et al. The polymorphic CAG repeat of the androgen receptor gene: a potential role in breast cancer in women over 40. Breast Cancer Res. Treat. 70, 109-116 (2001).

12. Dunning, A. et al. A systematic review of genetic polymorphisms and breast cancer risk. Cancer Epidemiol. Biomark. Prevent. 8, 843-854 (1999).

Program in Cancer Genetics

McGill University

Montreal, Quebec, Canada

Email:william.foulkes@mcgill.ca

\title{
Weak mitochondria permit protein clumps in Down syndrome
}

A distinct feature of Down syndrome (DS), or trisomy 21, is the onset of Alzheimer disease (AD) by middle age. Previous studies have suggested that increased expression of the gene encoding the amyloid $\beta$ precursor protein (A $\beta$ PP) may underlie the development of $A D$ in DS. However, DS individuals also have mitochondrial defects, which have been associated with neurodegeneration in other disorders. In a recent report, Busciglio et al. (Neuron 33, 677-688; 2002) draw the link between A $\beta-P P$ metabolism and mitochondrial dysfunction in DS.

Shown here are human astrocytes in cell culture stained with propidium iodide (red)
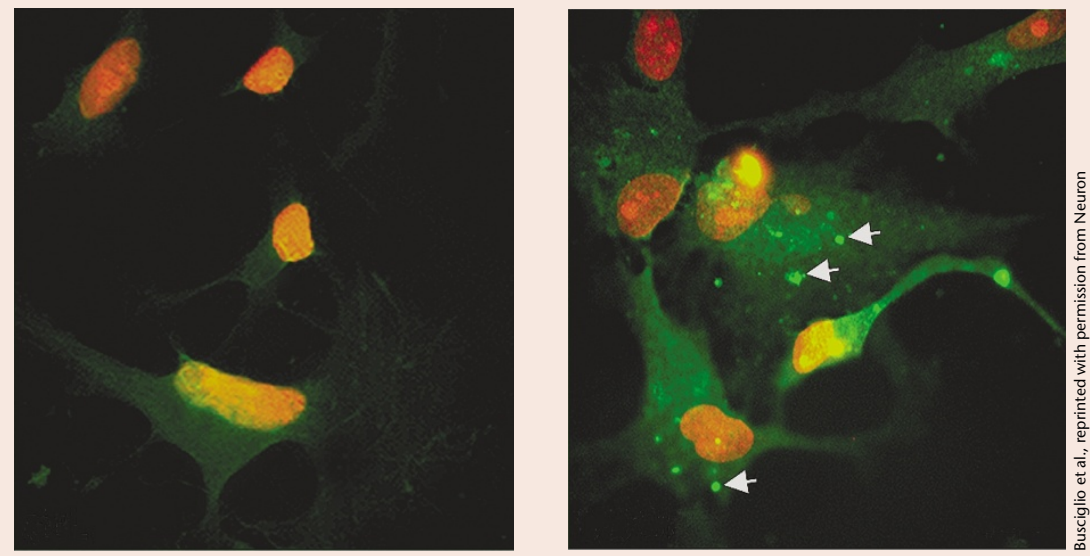
and an antibody that recognizes $A \beta-42$ (green), a product of A $\beta$ PP. Intracellular accumulation of A $\beta-42$ has been associated with AD in previous studies. The authors found that astrocytes treated with a mitochondrial inhibitor, m-chlorophynhydrazone (CCCP) also accumulated A $3-42$ (arrows; right). Untreated astrocytes did not accumulate A $\beta-42$ (left). The accumulation is similar to that seen in astrocytes derived from the brains of DS individuals, indicating that mitochondrial impairment may underlie the $A \beta-42$ defect in vivo.

Busciglio et al. went on to demonstrate that mitochondrial inhibition mimicked other alterations in A $\beta$-PP processing that occur in DS. Secreted A $\beta P P$, for example, was found to be reduced in both DS neurons and mitochondria-inhibited neurons. As secreted A $\beta$-PP promoted survival of DS neurons in vitro, its reduction could lead to neurodegeneration. The authors propose that impaired energy metabolism in DS neurons leads to alterations in the processing of A $\beta$-PP. Chronic A $\beta$-PP overexpression due to increased gene dosage could in turn impair mitochondrial function, they speculate, through direct toxicity or because of the metabolic costs of clearing aggregated proteins. 